\title{
The world of targeted therapies in kidney cancers: pitfalls, tips and tricks
}

\author{
This article was published in the following Dove Press journal: \\ OncoTargets and Therapy \\ 3 March 2017 \\ Number of times this article has been viewed
}

\author{
Alexis Vallard \\ Jane-Chloé Trone \\ Julien Langrand-Escure \\ Sophie Espenel \\ Jean-Baptiste Guy \\ Chloé Rancoule \\ Yaoxiong Xia \\ Anis El Meddeb Hamrouni \\ Majed Ben Mrad \\ Nicolas Magné
}

Department of Radiation Oncology, Lucien Neuwirth Cancer Institute,

Saint-Priest en Jarez, France
Correspondence: Nicolas Magné Department of Radiation Oncology, Lucien Neuwirth Cancer Institute, 108 Bis, Avenue Albert Raimond, BP 60008 , 4227I Saint-Priest en Jarez, France

$\mathrm{Tel}+334779$ I 7434

Fax +334779I 7197

Email nicolas.magne@icloire.fr

\begin{abstract}
In the past few years, metastatic renal cell carcinoma prognosis was improved by the development of molecular targeted therapies (TTs). At the metastatic stage, the tolerance to treatment is a major concern, not only because of the challenge of the efficacy/toxicity ratio improvement but also because of the importance of an optimal adherence to oral treatments. The present case series relates the issues of dealing with uncommon and sometimes never described side effects of sunitinib and sorafenib. The first case report deals with grade 3 vomiting during hemodialysis with concurrent administration of sunitinib. The second case is an iterative gout attack induced by sunitinib. The third case presents a grade 3 scalp dysesthesia with sorafenib. The fourth case includes an astonishing efficacy of metronomic (ie, low doses during a long period of time) bevacizumab in monotherapy. Multidisciplinary management and systematic reporting of unexpected efficacies and toxicities are needed to better understand TTs real therapeutic index. Although TTs revolutionized metastatic renal cell cancer prognosis, they also brought about previously unknown side effects. Identification and management of these off-target effects may be tricky, and therefore, comedication must be wisely chosen. As the physiopathology of these side effects is still unclear, multidisciplinary management and systematic reporting of toxicities are essential.
\end{abstract}

Keywords: renal cell carcinoma, bevacizumab, sunitinib, sorafenib, toxicity, efficacy

\section{Introduction}

Renal cell carcinoma ( $\mathrm{RCC}$ ) has the most dismal prognosis of all urinary tract cancers, and its incidence has been continuously rising over the past few years. ${ }^{1}$ It is estimated that $20 \%-30 \%$ of RCC patients are initially diagnosed with metastases, and that another $20 \%$ will present with metastatic recurrences. ${ }^{2}$ Prognosis of metastatic RCC was profoundly improved since 2006 with the development of targeted therapies (TTs) such as monoclonal antibodies, mTor inhibitors and tyrosine kinase inhibitors. TT almost doubled progression-free survival, ${ }^{3-5}$ and their successive use is currently recommended for the treatment of metastatic RCC. ${ }^{6}$ However, major questions regarding TT management are still unresolved, which include the optimal sequence of treatment, the potentiation of toxicities by comedication and the management of side effects. ${ }^{7}$ Considering the overall concept of efficacy/toxicity ratio, every adverse event has to be reported.

Sunitinib is a tyrosine kinase inhibitor targeting both vascular endothelial growth factor receptor (VEGFR) and platelet-derived growth factor receptor (PDGFR). The main reported off-target effects (grade $>2$ ) with sunitinib are fatigue, diarrhea, handfoot reaction, hypertension, peripheral edema, weight loss, dehydration, cough and hematologic toxicities. ${ }^{8-10}$ It is a standard of care in first-line treatment of metastatic kidney cancer. ${ }^{3,4,11,12}$ Sorafenib is a widely used multikinase inhibitor that blocks 
VEGFR, PDGFR and the RAF/MEK/ERK pathway, based on the RAF serine/threonine kinases hindrance. The main reported off-target effects (grade $>2$ ) with sorafenib include rash/desquamation, hypertension, hand-foot reaction and diarrhea. ${ }^{10,13}$ It is a possible option in second-line treatment of metastatic RCC. ${ }^{14}$ Bevacizumab is an antiangiogenic monoclonal antibody targeting isoforms of vascular endothelial growth factor A (VEGF-A) and is the first approved therapeutic agent to specifically target the tumor-associated vessels of solid tumors. ${ }^{15}$ It is currently recommended in association with interferon in first-line treatment and is mainly given in the case of indolent disease. ${ }^{14,16}$ As they target ubiquitous molecular pathways, TTs are known to sometimes induce unexpected complications, especially among aged patients. ${ }^{9,13}$ The identification and management of such side effects can be truly challenging. It is yet a point of primary interest since the quality of life of metastatic patient is the objective of major treatments, which can be ruined by rare and unpredictable toxicities.

We report here astonishing toxicities with the use of sunitinib and sorafenib, and a very long clinical efficacy of low-dose bevacizumab. Presented cases were chosen for their rarity, their originality, and the fact that causality was well established (exposure-withdrawal-re-exposure when possible). All toxicities were graded using the Common Terminology Criteria for Adverse Events v4.0. The institutional review board (Lucien Neuwirth Institute-CHU Ethic committee; Approval Number: 2012-00378) approved the study. All patients provided written consent.

\section{Short reports}

\section{Patient I: sunitinib-induced vomiting during hemodialysis sessions}

In 2004, a 66-year-old man was diagnosed with a papillary grade 2 nonmetastatic right renal cancer and was treated with radical right nephrectomy. A nonspecific $10 \mathrm{~mm}-$ diameter tumor was detected on his left kidney, and hence, he was placed under close surveillance. In 2005, a partial left nephrectomy was performed but no pathological signs of malignancy could be evidenced. In 2006, the patient was diagnosed with a prostatic adenocarcinoma, and hence, a radical prostatectomy was performed without any complication. A few months later, multiple suspect left renal lesions were identified and a left radical nephrectomy was recommended by the multidisciplinary urologic board after meeting. Pathological analyses revealed a papillary renal cancer. The patient underwent hemodialysis 3 days a week (Monday, Wednesday and Friday) after bilateral complete nephrectomy and was closely monitored. Hemodialysis was performed between 2007 and 2010 without any complications. In 2010, a $70 \mathrm{~mm}$ local recurrence was diagnosed in the left renal fossa bed. Biopsies were performed, evidencing a recurrence of the papillary renal cancer. Since complete resection was impossible and in agreement with patient's nephrologists, sunitinib was initiated in August 2010. For the first cycle, the smallest recommended dose of sunitinib was administered (ie, $37.5 \mathrm{mg} /$ day). Sunitinib cycles consisted of 28 days on and 14 days off treatment. All along sunitinib administration, blood pressure was monitored weekly. He was considered to be stable and did not require treatment initiation.

Grade 3 vomiting occurred during all hemodialysis sessions performed following the first sunitinib initiation. Anticipatory nausea/vomiting was not suspected since the patient was not reported to be anxious and anxiolytic treatments did not decrease the symptoms. However, the 14 days treatment break was associated with a complete resolution of nausea and a related-to-sunitinib side effect was suspected.

Ondansetron was prescribed during the whole second cycle of sunitinib, but grade 1-2 vomiting during hemodialysis sessions was still reported. Digestive symptoms ceased again during the sunitinib off period.

Because of a grade 2 thrombocytopenia, the third cycle was delayed and the sunitinib dose was reduced to $25 \mathrm{mg} /$ day. Despite the dose reduction and ondansetron systematic administration, grade 1 vomiting under hemodialysis was systematic during the sunitinib administration. Vomiting only stopped after sunitinib was replaced with another TT because of disease progression after three sunitinib cycles.

\section{Patient 2: sunitinib-induced gout attack}

In 2008, a 69-year-old man was diagnosed with a nonmetastatic Fuhrman grade 3 left RCC. A radical left nephrectomy was performed, and the patient was monitored for 2 years without any other medical complications. No renal failure or electrolyte disorders were reported. In 2010, a metastatic progression was suspected on a systematic computed tomography scan, with lateral aortic nodes involvement and a left renal fossa tumor extended to the left colon and diaphragm. Sunitinib was initiated at $37.5 \mathrm{mg} /$ day after biopsies confirmed a histology-proven metastatic RCC recurrence. The patient was treated with a 28-day on and 14-day off schedule. Plasma uric acid was normal at baseline. During the first cycle of this treatment, the patient presented his very first episode of gout attack located on a big toe. As it was reported to be normal in $30 \%$ of acute gout attacks if 
assessed immediately, the plasma uric acid concentration was assessed 15 days later, which was $81 \mathrm{mg} / \mathrm{L}$ (normal plasma uric acid concentration $<70 \mathrm{mg} / \mathrm{L}$ ). No concomitant renal failure, hemoglobin and/or hematocrit disorder, or concurrent medication intake other than sunitinib (acetylsalicylic acid, diuretics, pyrazinamide, ethambutol, etc.) were reported. Purine-rich food and alcohol were cutoff, but the patient did not consume much of them. Allopurinol (100 mg/day) was initiated before the second cycle of sunitinib without colchicine because interactions with sunitinib were feared. Two months later, during the fourth sunitinib cycle, the patient developed a similar acute gout attack without any identified triggering factor. Allopurinol dose was increased to maximum (300 mg/day), while sunitinib was maintained at $37.5 \mathrm{mg} /$ day until progression. The patient kept sticking to his purine-depleted diet. Iterative gout attacks of lower intensity were still periodically reported during the following months under sunitinib therapy. After progression, a new line of treatment was initiated. Sunitinib discontinuation was followed by a total regression of gout attacks.

\section{Patient 3: scalp dysesthesia with sorafenib}

In 2010, a 68-year-old woman was diagnosed with an abdominal-metastatic grade 3 RCC. Following total nephrectomy, the patient was treated with sunitinib from March to June 2010. Due to poor hepatic tolerance and tumor progression, the patient was switched to everolimus. Unfortunately, in the months following the second-line treatment initiation, she was diagnosed with everolimusrelated bilateral interstitial pneumonia, requesting for urgent everolimus discontinuation. A third-line treatment based on sorafenib was initiated in January 2011. Despite immediate grade 1 abdominal, facial and truncal rash, sorafenib was continued at $200 \mathrm{mg}$ twice a day. Twelve days later, the patient reported grade 3 dysesthesia of the whole scalp, negatively affecting her quality of life. The differential diagnosis of migraine was rejected by neurologists, and first-line migraine treatments did not improve dysesthesia. Sorafenib was stopped 15 days after the onset of dysesthesia and when the symptoms intensified. Sorafenib discontinuation resulted in a complete dysesthesia resolution in the following days.

\section{Patient 4: long efficacy of low-dose bevacizumab}

In 1998, a radical nephrectomy was performed on a 79-yearold man with an intent to cure nonmetastatic grade 2 RCC. Histology-proven metastatic progression was diagnosed in 2006 , with a painful $50 \times 50 \mathrm{~mm}$ lytic lesion of the left scapula. A palliative radiotherapy was successfully performed (39 Gy, $3 \mathrm{~Gy} /$ fraction) with significant pain relief. Because of the patient's age, bevacizumab was initiated in monotherapy at a third of the recommended dose (ie, $5 \mathrm{mg} / \mathrm{kg}$ intravenously every 3 weeks instead of $10 \mathrm{mg} / \mathrm{kg}$ every 2 weeks). The patient was 88 years old at that time but was still in excellent condition. Bevacizumab could be continued for another 5 years with a perfect tolerance and good efficacy: Scapula metastasis was stable $(43 \times 38 \mathrm{~mm})$ and painless. Bevacizumab was stopped in 2011, considering the extended absence of cancer progression. Six months later, an isolated progression of the scapula lesion was reported, with an increasing left shoulder pain and an increasing metastasis volume $(50 \times 60 \mathrm{~mm})$. Bevacizumab was readministered in 2012 with a pain resolution, and tumor volume decreased in 9 weeks. To date, the patient is still alive with an excellent tolerance. Intermittent ("start-and-stop") bevacizumab is continued with a clear on/off effect on pain relief.

\section{Discussion}

If TT revolutionized the standard of care of metastatic renal cancer, the real therapeutic index is sometimes hard to define, with unpredictable toxicities as well as spectacular efficacies. The present clinical cases highlight the incredible, various side effects or benefits that seem hard to relate to a TT at first sight. However, knowledge of rare toxicities is essential since it can prevent oncologists from performing numerous unnecessary medical imaging or complementary investigation. Management of such complications must be reported since toxicities can impair patients' quality of life - and therefore, adherence to oral treatments - for long periods of time if they are misunderstood. Similarly, cases of surprising efficacy with perfect tolerance should be reported, as TT often represents the only reasonable treatment for elderly metastatic patient.

The most common side effects reported with sunitinib are fatigue (grades 3-4, 16.4\%), diarrhea (grade 3, 10.9\%), vomiting, hypertension (20\%), stomatitis and hand-foot syndrome (grade 3,9.1\%). ${ }^{17}$ Although the prevalence of RCC among patients with hemodialysis is 40-100 times higher than the general population, ${ }^{18}$ the use of sunitinib in dialysis patients is poorly described. Studies based on the limited number of patients seem to suggest that concomitant use of sunitinib and dialysis is feasible with good results. ${ }^{19-21}$ Furthermore, it was suggested that hemodialysis did not significantly alter plasma concentrations of sunitinib. $22-24$ Although other grade $\geq 3$ toxicities have been reported with concomitant dialysis and sunitinib (mainly hypertension), ${ }^{23,25}$ 
we report here, for the first time, related-to-dialysis-and-sun itinib vomiting. To date, no physiopathological explanation could be proposed to link the onset of iterative vomiting and dialysis.

Acute gout induced by sunitinib has rarely been reported, ${ }^{26}$ and sometimes probably as sunitinib was associated with concomitant anticancer drugs. ${ }^{27}$ The therapeutic management of gout induced by sunitinib should probably differ from "common" gout since important toxicities have been reported with the concomitant administration of colchicine and sunitinib. ${ }^{28}$ The relationship between the administration of sunitinib and increased plasma uric acid concentration is still poorly understood.

Most commonly reported cutaneous adverse effects of sorafenib were hand-foot skin reaction (grade 3, 30\%), which usually appeared to be early but might request a dose reduction. ${ }^{29,30}$ Scalp dysesthesia with sorafenib was already described with lower grades of intensity in previous studies. ${ }^{29}$ However, no physiological explanation of this side effect could be found.

Outcomes of a large phase 3 trial were published in 2016 in the Lancet journal, and the article extensively reported toxicities from 625 patients treated with sunitinib and 628 patients treated with sorafenib. ${ }^{10}$ However, the toxicities that are described in our article were not reported in this publication: Only 14 cases of vomiting ( $2 \%$ of sunitinib patients) were reported in the Lancet trial but none during hemodialysis, no gout attack was reported (and hyperuricemia was not assessed), no neuropathic pain was reported with sorafenib, and scalp pain (which differs from dysesthesia) was reported in two patients $(<1 \%)$. Moreover, the phase 3 trials are not designed to prove the drug causality of a clinical event. In the present report, treatment discontinuation and reintroduction were sometimes performed, adding value to our observation that describes in detail toxicities that have rarely or never been reported.

For each last case, the burning question is undoubtedly: How to optimally manage unexplainable side effects and how to come to the decision to continue or discontinue the targeted treatment? Concomitant treatment, such as that with colchicine, might be contraindicated, and the balance between toxicity and efficacy should always be evaluated. The exhaustive reporting of related-to-TT toxicities is a point of primary interest, which might help oncologists to deal with these major topics. Multidisciplinary interactions are probably needed. No precise molecular explanation and no potential solution were found in the literature, indeed. As these reported off-target effects are exceptional, none have been specifically studied.
For instance, the gout attack physiopathology includes a direct participation of the intra-articular Src tyrosine kinases, activating caspase 1 through NLPR3 inflammasome, finally leading to the production of inflammatory cytokines and chemokines that are responsible for the clinical symptoms. ${ }^{31}$ The impact of sunitinib on Src tyrosine kinase is still debated, ${ }^{32,33}$ but the explanation of sunitinib-related side effects is certainly to be found in its multi-kinase activities that were well described by Karaman et al. ${ }^{34}$ Another physiological explanation for these unexpected toxicities might be the age of the involved patients, since all were $>65$ years old. In aged patients, the simultaneous alteration of the pharmacokinetics and the pharmacodynamics tends to increase anticancer drug toxicities. Pharmacokinetics phenomena (involving an altered drug distribution) extend the plasma exposure in aged patients with a major impact of the lean muscle mass loss, which was well described with sorafenib-induced toxicities. ${ }^{35}$ Weekly assessment of sorafenib in plasma of aged patients revealed that sorafenib concentrations could vary at a ratio of 1:2 considering sarcopenic versus non-sarcopenic patients. ${ }^{36}$ Therefore, TTs should be administered with caution in oldest patients, sometimes with modified protocols (for instance, 2 weeks on, 1 week off, with sunitinib). But above all, these patients should be included in geriatric oncology trials, and guaranteed the most precocious and the best possible oncogeriatric management. Trials are currently developed and are recruiting, particularly with the Geriatric Oncology Group (with trials depending on the primary tumor location), and the International Society of Geriatric Oncology and Onco-Geriatric Francophone Society groups (with interdisciplinary programs). There is, for the moment, no consensus regarding geriatric population-adapted treatments, which makes any therapeutic decision difficult. If the use of oncogeriatric scales influences the final therapeutic decision, it does not define adapted chemotherapy programs. ${ }^{37}$ Therefore, reporting the contribution of real-life toxicity and efficacy in the heterogeneous population of elderly patients receiving a TT is an absolute necessity.

Bevacizumab monotherapy at low dose demonstrated, in the present case report, a good efficacy with an excellent tolerance. If it was initially thought that new molecular TTs would make bevacizumab disappear, the present case highlights the fact that it can still be in 2016 a useful drug in the treatment of metastatic RCC. ${ }^{38}$ The "on/off" effect of the bevacizumab in bone metastasis was also highlighted. ${ }^{39}$ Because of its tumor-vessels addiction and other previously described angiogenic mechanisms, metastatic RCC is probably a situation where metronomic (ie, low doses for long periods of time) antiangiogenic agents may induce interesting responses. ${ }^{40,41}$ 
As reported, it might also be a surprisingly well-tolerated therapy for elderly patients, and low-dose bevacizumab in monotherapy may be an interesting option when recommended anticancer drugs cannot be administered.

\section{Conclusion}

Over the past decade, numerous TTs were developed, sometimes with conventional side effects and sometimes with unusual ones. Oncologists should be able to make the connection between unexpected toxicities and TT, although the physiopathological mechanisms are often unknown. Multidisciplinary management and systematic reporting are essential to better understand and prevent these toxicities. The efficacy/toxicity ratio should guide oncologists' choices, considering that TT can also sometimes offer a real therapeutic opportunity for aged patients.

\section{Disclosure}

The authors report no conflicts of interest in this work.

\section{References}

1. Rini BI, Campbell SC, Escudier B. Renal cell carcinoma. Lancet. 2009;373(9669):1119-1132.

2. Ljungberg B, Campbell SC, Choi HY, et al. The epidemiology of renal cell carcinoma. Eur Urol. 2011;60(4):615-621.

3. Motzer RJ, Hutson TE, Tomczak P, et al. Overall survival and updated results for sunitinib compared with interferon alpha in patients with metastatic renal cell carcinoma. J Clin Oncol. 2009;27(22):3584-3590.

4. Escudier B, Eisen T, Stadler WM, et al; TARGET Study Group. Sorafenib in advanced clear-cell renal-cell carcinoma. NEngl J Med. 2007;356(2): 125-134.

5. Motzer RJ, Escudier B, Oudard S, et al; RECORD-1 Study Group. Phase 3 trial of everolimus for metastatic renal cell carcinoma: final results and analysis of prognostic factors. Cancer. 2010;116(18):4256-4265.

6. Escudier B, Porta C, Schmidinger M, et al; ESMO Guidelines Working Group. Renal cell carcinoma: ESMO Clinical Practice Guidelines for diagnosis, treatment and follow-up. Ann Oncol. 2014;25(Suppl 3): iii49-iii56.

7. Langrand-Escure J, Vallard A, Rivoirard R, et al. Safety assessment of molecular targeted therapies in association with radiotherapy in metastatic renal cell carcinoma: a real-life report. Anticancer Drugs. 2016; 27(5):427-432.

8. Gore ME, Szczylik C, Porta C, et al. Safety and efficacy of sunitinib for metastatic renal-cell carcinoma: an expanded-access trial. Lancet Oncol. 2009;10(8):757-763.

9. Hutson TE, Bukowski RM, Rini BI, et al. Efficacy and safety of sunitinib in elderly patients with metastatic renal cell carcinoma. Br J Cancer. 2014;110(5):1125-1132.

10. Haas NB, Manola J, Uzzo RG, et al. Adjuvant sunitinib or sorafenib for high-risk, non-metastatic renal-cell carcinoma (ECOG-ACRIN E2805): a double-blind, placebo-controlled, randomised, phase 3 trial. Lancet 2016;387(10032):2008-2016.

11. Motzer RJ, Rini BI, Bukowski RM, et al. Sunitinib in patients with metastatic renal cell carcinoma. JAMA. 2006;295(21):2516-2524.

12. Armstrong AJ, Halabi S, Eisen T, et al. Everolimus versus sunitinib for patients with metastatic non-clear cell renal cell carcinoma (ASPEN): a multicentre, open-label, randomised phase 2 trial. Lancet Oncol. 2016; 17(3):378-388.
13. Procopio G, Bellmunt J, Dutcher J, et al. Sorafenib tolerability in elderly patients with advanced renal cell carcinoma: results from a large pooled analysis. Br J Cancer. 2013;108(2):311-318.

14. Patard JJ, Baumert H, Bensalah K, et al; Les membres du CCAFU. [CCAFU Recommendations 2013: Renal cancer]. Prog Urol. 2013; 23(Suppl 2):S177-S204. French [with English abstract].

15. Ferrara N, Hillan KJ, Gerber HP, Novotny W. Discovery and development of bevacizumab, an anti-VEGF antibody for treating cancer. Nat Rev Drug Discov. 2004;3(5):391-400.

16. Molina AM, Motzer RJ, Heng DY. Systemic treatment options for untreated patients with metastatic clear cell renal cancer. Semin Oncol. 2013;40(4):436-443.

17. Motzer RJ, Hutson TE, Tomczak P, et al. Sunitinib versus interferon alfa in metastatic renal-cell carcinoma. $N$ Engl J Med. 2007;356(2): 115-124.

18. Maisonneuve $\mathrm{P}$, Agodoa L, Gellert R, et al. Cancer in patients on dialysis for end-stage renal disease: an international collaborative study. Lancet. 1999;354(9173):93-99.

19. Czarnecka AM, Kawecki M, Lian F, Korniluk J, Szczylik C. Feasibility, efficacy and safety of tyrosine kinase inhibitor treatment in hemodialyzed patients with renal cell cancer: 10 years of experience. Future Oncol. 2015;11(16):2267-2282.

20. Josephs D, Hutson TE, Cowey CL, et al. Efficacy and toxicity of sunitinib in patients with metastatic renal cell carcinoma with severe renal impairment or on haemodialysis. BJU Int. 2011;108(8):1279-1283.

21. Park S, Lee J, Park SH, et al. Treatment of hemodialyzed patients with sunitinib in renal cell carcinoma. Chemotherapy. 2010;56(6):485-491.

22. Masini C, Sabbatini R, Porta C, et al. Use of tyrosine kinase inhibitors in patients with metastatic kidney cancer receiving haemodialysis: a retrospective Italian survey. BJU Int. 2012;110(5):692-698.

23. Vickers MM, Heng DY, Hemmelgarn B, Eigl BJ. Tolerance of sunitinib in dialyzed patients with metastatic renal cell carcinoma. Clin Genitourin Cancer. 2009;7(3):E104-E106.

24. Izzedine H, Etienne-Grimaldi MC, Renée N, Vignot S, Milano G. Pharmacokinetics of sunitinib in hemodialysis. Ann Oncol. 2009;20(1): 190-192.

25. Yildiz I, Sen F, Kilic L, Ciftci R, Basaran M. Intolerance to sunitinib treatment in hemodialysis patients with metastatic renal cell carcinoma. Korean J Urol. 2014;55(1):74-76.

26. Li XS, Wu X, Zhao PJ, et al. Efficacy and safety of sunitinib in the treatment of metastatic renal cell carcinoma. Chin Med J (Engl). 2011; 124(18):2920-2924

27. Patel PH, Senico PL, Curiel RE, Motzer RJ. Phase I study combining treatment with temsirolimus and sunitinib malate in patients with advanced renal cell carcinoma. Clin Genitourin Cancer. 2009;7(1): 24-27.

28. Abodunde OA, LevakaVeera RR, Desai R, Nweke N, Berrou M. Colchicine toxicity precipitated by interaction with sunitinib. J Clin Pharm Ther. 2013;38(3):243-245.

29. Autier J, Escudier B, Wechsler J, Spatz A, Robert C. Prospective study of the cutaneous adverse effects of sorafenib, a novel multikinase inhibitor. Arch Dermatol. 2008;144(7):886-892.

30. Lee WJ, Lee JL, Chang SE, et al. Cutaneous adverse effects in patients treated with the multitargeted kinase inhibitors sorafenib and sunitinib. Br J Dermatol. 2009;161(5):1045-1051.

31. Gout attack: physiopathology, diagnosis, treatment, evolution. Paris: Bichat University Hospital, Pr Meyer [updated 2011]. Available from: http://www.rhumatologie-bichat.com/Cours\%20D3\%20Goutte. pdf. Article in French.

32. Zhang $\mathrm{K}$, Wang $\mathrm{X}$, Wang $\mathrm{H}$. Effect and mechanism of Src tyrosine kinase inhibitor sunitinib on the drug-resistance reversal of human A549/DDP cisplatin-resistant lung cancer cell line. Mol Med Rep. 2014; 10(4):2065-2072.

33. Bai L, Yang JC, Ok JH, Mack PC, Kung HJ, Evans CP. Simultaneous targeting of Src kinase and receptor tyrosine kinase results in synergistic inhibition of renal cell carcinoma proliferation and migration. Int $J$ Cancer. 2012;130(11):2693-2702. 
34. Karaman MW, Herrgard S, Treiber DK, et al. A quantitative analysis of kinase inhibitor selectivity. Nat Biotechnol. 2008;26(1):127-132.

35. Antoun S, Baracos VE, Birdsell L, Escudier B, Sawyer MB. Low body mass index and sarcopenia associated with dose-limiting toxicity of sorafenib in patients with renal cell carcinoma. Ann Oncol. 2010;21(8): 1594-1598.

36. Mir O, Coriat R, Blanchet B, et al. Sarcopenia predicts early doselimiting toxicities and pharmacokinetics of sorafenib in patients with hepatocellular carcinoma. PLoS One. 2012;7(5):e37563.

37. Chaïbi $P$, Magné $N$, Breton $S$, et al. Influence of geriatric consultation with comprehensive geriatric assessment on final therapeutic decision in elderly cancer patients. Crit Rev Oncol Hematol. 2011;79(3): 302-307.

38. Guillot A, Levy A, Pacaut C, et al. Reappraisal of the role of bevacizumab in the therapeutic strategy in advanced renal cell carcinoma. Clin Genitourin Cancer. 2012;10(3):147-152.
39. Mazeron R, Anderson B, Supiot S, Paris F, Deutsch E. Current state of knowledge regarding the use of antiangiogenic agents with radiation therapy. Cancer Treat Rev. 2011;37(6):476-486.

40. de Castro Junior G, Puglisi F, de Azambuja E, El Saghir NS, Awada A. Angiogenesis and cancer: a cross-talk between basic science and clinical trials (the "do ut des" paradigm). Crit Rev Oncol Hematol. 2006; 59(1):40-50.

41. Melichar B, Bracarda S, Matveev V, et al; BEVLiN Investigators. A multinational phase II trial of bevacizumab with low-dose interferon$\alpha 2 \mathrm{a}$ as first-line treatment of metastatic renal cell carcinoma: BEVLiN. Ann Oncol. 2013;24(9):2396-2402.
OncoTargets and Therapy

\section{Publish your work in this journal}

OncoTargets and Therapy is an international, peer-reviewed, open access journal focusing on the pathological basis of all cancers, potential targets for therapy and treatment protocols employed to improve the management of cancer patients. The journal also focuses on the impact of management programs and new therapeutic agents and protocols on

\section{Dovepress}

patient perspectives such as quality of life, adherence and satisfaction. The manuscript management system is completely online and includes a very quick and fair peer-review system, which is all easy to use. Visit http://www.dovepress.com/testimonials.php to read real quotes from published authors. 